\title{
Subcutaneous Dermatofibroma
}

\author{
Kyu Dong Jung, M.D., Dong-Youn Lee, M.D., Joo-Heung Lee, M.D., Jun-Mo Yang, M.D., \\ Eil-Soo Lee, M.D.
}

Department of Dermatology, Samsung Medical Center, Sungkyunkwan University School of Medicine, Seoul, Korea

\begin{abstract}
Dermatofibroma (DF) is usually confined to the dermis and the overlying epidermis is usually hyperplastic. Although DF with deep subcutaneous extension is commonly encountered, purely subcutaneous DF is uncommon. In this review, we describe a case of a 41-year-old male patient who presented with a painless, subcutaneous, hard papule on the left thigh. After the skin had been incised the lesion was totally removed, and histopathology revealed a subcutaneous dermatofibroma. (Ann Dermatol 23(2) 254 257, 2011)
\end{abstract}

\section{-Keywords-}

Dermatofibroma, Fibrous histiocytoma, Subcutaneous

\section{INTRODUCTION}

Dermatofibroma (DF) is perhaps the most common mesenchymal growth of the skin. It has a predilection for the lower legs of women in their early 20s. This tumor most frequently occurs in the dermis with or without extending into the subcutaneous tissue ${ }^{1}$. The benign DF is usually confined to the dermis, whereas the occurrence of this lesion purely in the subcutaneous tissue has uncommonly been reported ${ }^{2-5}$. According to the $\mathrm{WHO}$, a deep location is found for less than $1 \%$ of all benign fibrous histiocytomas $^{5}$. Herein, we report an uncommon case of a purely subcutaneous DF.

Received May 13, 2010, Revised July 8, 2010, Accepted for publication July 8, 2010

Corresponding author: Eil-Soo Lee, M.D., Department of Dermatology, Samsung Medical Center, Sungkyunkwan University School of Medicine, 50 Irwon-dong, Gangnam-gu, Seoul 135-710, Korea. Tel: 82-2-3410-6578, Fax: 82-2-3410-3869, E-mail: es4603.lee@samsung. com

\section{CASE REPORT}

A 41-year-old male presented to our dermatology outpatient clinic, with an apparently asymptomatic palpable subcutaneous skin lesion on the left thigh, appearing 3 months prior to his visit to our clinic. There was no previous history of medication use or trauma and no family history of skin disease. The lesion was a hard, solitary, discrete, subcutaneous papule. The lesion had not been increasing in size over the last 3 months. The hard subcutaneous papule popped out when an incision for biopsy was made into the skin above the lesion. Histologic findings showed a well-circumscribed, subcutaneous papule composed of fibrohistiocytic proliferation (Fig. 1A). Storiform arrangement of spindle cells, trapping normal collagen bundles at the periphery of the lesion were consistent with subcutaneous DF (Fig. 1B). There was no nuclear pleomorphism or hyperchromasia (Fig. 1C). Immunohistochemical staining demonstrated positivity for factor XIIla (polyclonal, dilution 1:200, Biogenex, Fremont, CA, USA) (Fig. 2A), and negativity for CD34 (monoclonal, clone QBEND 10 dilution 1:50, Dako, Carpinteria, CA, USA) (Fig. 2B), desmin (monoclonal, Clone D33 dilution 1:20, Dako) (Fig. 2C). CD68 (monoclonal, clonePG-M1 dilution 1:50, Dako) staining showed negative reactions in the spindle cells (Fig. 2D). Microscopic and immunohistochemical examinations confirmed the diagnosis of subcutaneous DF. The patient is asymptomatic with no evidence of recurrent disease at the 3-year follow-up.

\section{DISCUSSION}

Our case showed strong immunohistochemical positivity for factor XIIla (polyclonal, dilution 1:200, Biogenex) and negativity for CD34 (monoclonal, clone QBEND 10 dilution 1:50, Dako), desmin (monoclonal, Clone D33 dilution 1:20, Dako) and CD68 (monoclonal, clonePG-M1 


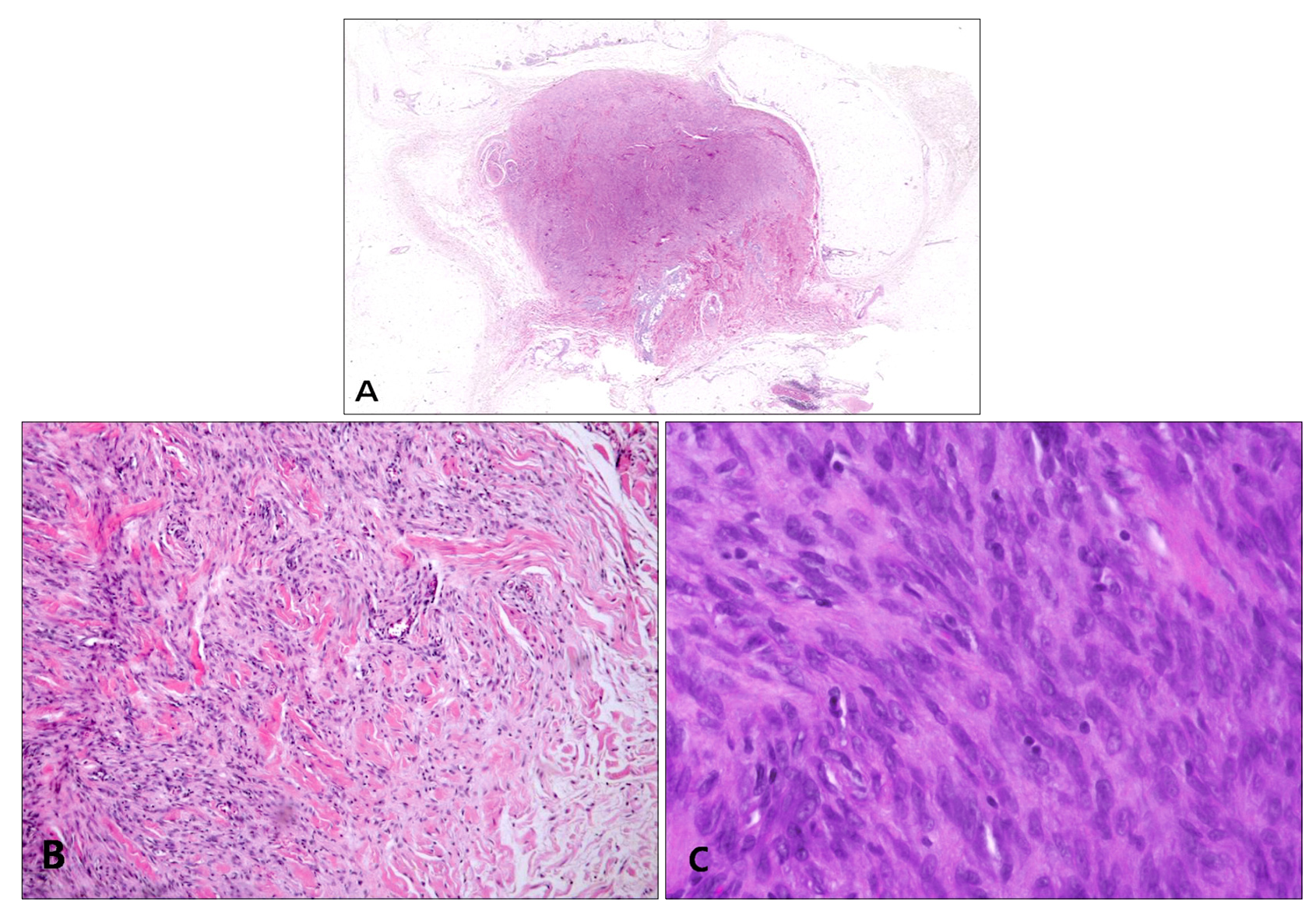

Fig. 1. Incisional biopsy specimen shows well-circumscribed, subcutaneous nodule $(A: H \& E, \times 1)$. The tumor cells are arranged in storiform pattern and are trapping normal collagen bundles at the periphery of the tumor nodule (B: H\&E, $\times 100)$. In higher magnification, tumor cells are cytologically bland and are spindle-shaped with variable amounts of pale eosinophilic, ill-defined cytoplasm. Nuclei are elongated and histiocyte-like with vesicular chromatin pattern. There is no nuclear pleomorphism or hyperchromasia (C: H\&E, $\times 400)$.

dilution 1:50, Dako) staining showed negative reactions in the spindle cells.

It is important to recognize the pure subcutaneous variant of DF because it may be confused with a variety of benign and malignant subcutaneous tumors ${ }^{6}$. Among the benign subcutaneous tumors, deep penetrating dermatofibroma (DPDF) or dermatofibroma extending into the subcutaneous tissue was described as a variant of dermatofibroma by Kamino and Jacobson ${ }^{7}$ in 1990. Kamino and Jacobson ${ }^{7}$ published the histologic criteria to distinguish DPDF from dermatofibrosarcoma protuberans (DFSP). Scanning magnification is the most helpful tool in distinguishing a regular, smooth, nodular or scalloped pattern of demarcation in DPDF from the irregular, lacelike/honeycomb or multilayered pattern of DFSP ${ }^{8}$. In previous reports, most cases of DPDF were positive for factor XIIla and negative for CD34 and vice versa in cases of DFSP.

Malignant fibrohistiocytic neoplasms are composed of malignant pleomorphic sarcomatous cells, bizarre giant cells, and frequent mitotic figures. Histological features, unfortunately, play a minor role in predicting the biologic behavior of these neoplasms ${ }^{9}$. The presence of certain atypical histological features, including necrosis, marked cellular and mitotic activity, does not correlate well with clinical recurrence.

As with benign fibrohistiocytic neoplasms, the diagnosis of malignant fibrohistiocytic neoplasms frequently relies upon electron microscopy and immunohistochemistry to differentiate it from other lesions. Like DF, DFSP occurs in the dermis and subcutis, but is more apt to show extensive subcutaneous involvement than benign DF. It is also characterized by a more uniform cellular population and lacks giant cells, inflammatory cells, and xanthomatous elements. Its fascicles, composed of slender attenuated cells, are longer and arranged in a distinct storiform pattern, unlike the short curlicue fascicles of DF. Its margins are infiltrative in contrast to the well-defined margins of $\mathrm{DF}^{10,11}$. However, DFSP may be difficult to differentiate 

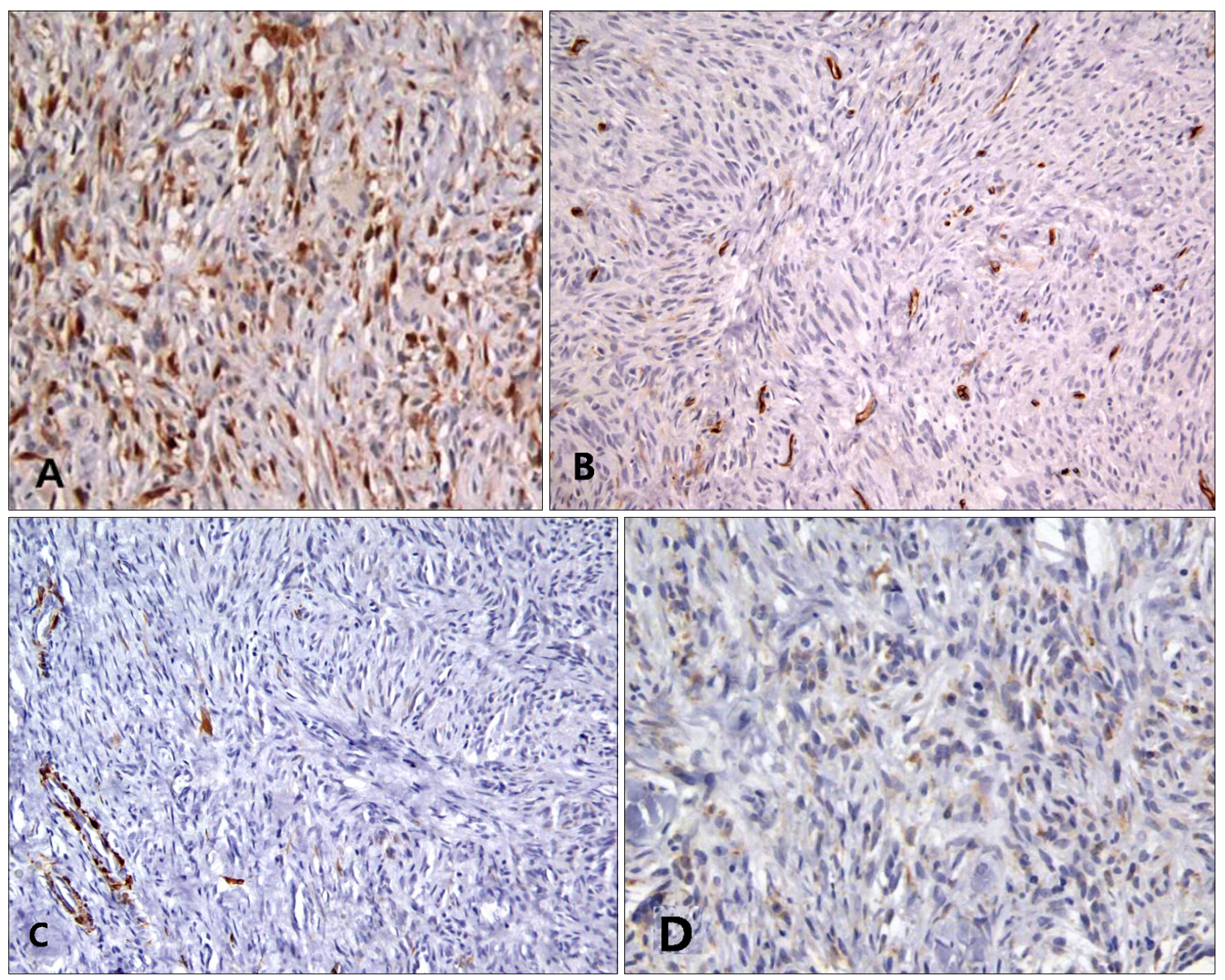

Fig. 2. On the immunohistochemical staining, there are positive reactions for Factor XIIla and negative reaction for CD34 and desmin in the spindle cells (A: Factor XIIla, $\times 200, B$ : CD34, $\times 200$, C: desmin, $\times 200$ ). The CD68 staining shows negative reactions in the spindle cells (D: CD68, ×200).

from DF. Immunostaining can help to reveal the distinct differences in the cellular composition of these tumors. DF contains a significant population of factor XIIla-positive cells, although it has been debated whether these cells represent a population of neoplastic cells or a peculiar infiltrate that accompanies the tumor. In contrast, DFSP contains only scattered factor XIIla-positive cells, but, in striking contrast to DF, it expresses CD34 in a significant portion of neoplastic cells ${ }^{12,13}$. The combination of these two stains has proved to be highly reliable in distinguishing these two lesions, which often cause diagnostic problems, particularly when only a superficial biopsy specimen is available for assessment.

Usually, DF does not require treatment unless it is symptomatic. As per the literature, most subcutaneous DF's were treated by local excision without sacrificing structures that would cause major functional or cosmetic morbidity. Our patient had not symptom but the lesion was removed when an incision for biopsy was made into the skin above the lesion.

DF almost pursues a benign course, with a recurrence rate of less than $2 \%$, although a higher rate of recurrence has been reported for some variants, including the cellular, aneurysmal, and atypical (pseudosarcomatous) forms ${ }^{14}$. A higher rate of recurrence has been noted in the subcutaneous and deep types and in lesions located on the face, in which a recurrence rate of $15 \%$ has been reported $^{6,15}$. Also, a local recurrence rate of $19 \%$ (four of 21 cases) has been reported in 21 cases from the previous subcutaneous DF series ${ }^{2}$. Accordingly, if the lesion has been proved to be subcutaneous DF with or without symptoms, it would seem reasonable to recommend that the lesion should be completely removed by local excision. In this paper, we have reported an uncommon case of 
subcutaneous DF.

\section{REFERENCES}

1. Meister P, Konrad E, Krauss F. Fibrous histiocytoma: a histological and statistical analysis of 155 cases. Pathol Res Pract 1978;162:361-379.

2. Fletcher CD. Benign fibrous histiocytoma of subcutaneous and deep soft tissue: a clinicopathologic analysis of 21 cases. Am J Surg Pathol 1990;14:801-809.

3. Chang SE, Choi JH, Sung KJ, Moon KC, Koh JK. Subcutaneous dermatofibroma showing a depressed surface. Int J Dermatol 2001;40:77-78.

4. Skoulakis CE, Papadakis CE, Datseris GE, Drivas El, Kyrmizakis DE, Bizakis JG. Subcutaneous benign fibrous histiocytoma of the cheek. Case report and review of the literature. Acta Otorhinolaryngol Ital 2007;27:90-93.

5. Garrido-Ruiz MC, Ramos $P$, Enguita $A B$, Rodriguez Peralto JL. Subcutaneous atypical fibrous histiocytoma. Am J Dermatopathol 2009;31:499-501.

6. Mentzel $T$, Kutzner $H$, Rütten $A$, Hügel $H$. Benign fibrous histiocytoma (dermatofibroma) of the face: clinicopathologic and immunohistochemical study of 34 cases associated with an aggressive clinical course. Am J Dermatopathol 2001; 23:419-426.

7. Kamino $\mathrm{H}$, Jacobson M. Dermatofibroma extending into the subcutaneous tissue. Differential diagnosis from dermatofibrosarcoma protuberans. Am J Surg Pathol 1990;14:1156-
1164.

8. Zelger B, Sidoroff A, Stanzl U, Fritsch PO, Ofner D, Zelger $B$, et al. Deep penetrating dermatofibroma versus dermatofibrosarcoma protuberans. A clinicopathologic comparison. Am J Surg Pathol 1994;18:677-686.

9. Enzinger FM, Weiss SW. Malignant fibrous histiocytoma (pleomorphic undifferentiated sarcoma). In: Weiss SW, Goldblum JR, editors. Enzinger and Weiss' soft tissue tumors. 5th ed. Philadelphia: Elsevier Mosby, 2008:403-428.

10. Aiba S, Tabata N, Ishii H, Ootani H, Tagami H. Dermatofibrosarcoma protuberans is a unique fibrohistiocytic tumour expressing CD34. Br J Dermatol 1992;127:79-84.

11. Chen TC, Kuo T, Chan HL. Dermatofibroma is a clonal proliferative disease. J Cutan Pathol 2000;27:36-39.

12. Kamino H, Salcedo E. Histopathologic and immunohistochemical diagnosis of benign and malignant fibrous and fibrohistiocytic tumors of the skin. Dermatol Clin 1999;17: 487-505.

13. Nakanishi S, Shinomiya S, Sano T, Hizawa K. Immunohistochemical observation of intracytoplasmic lysozyme in proliferative and neoplastic fibrohistiocytic lesions. Acta Pathol Jpn 1982;32:949-959.

14. Bisceglia M, Attino V, Bacchi CE. Metastasizing "benign" fibrous histiocytoma of the skin: a report of two additional cases and review of the literature. Adv Anat Pathol 2006;13:89-96.

15. Colome-Grimmer MI, Evans HL. Metastasizing cellular dermatofibroma. A report of two cases. Am J Surg Pathol 1996;20:1361-1367. 